\title{
The significance and robustness of a plasma free amino acid (PFAA) profile-based multiplex function for detecting lung cancer
}

Masato Shingyoji ${ }^{1 *}$, Toshihiko lizasa ${ }^{1}$, Masahiko Higashiyama ${ }^{2}$, Fumio Imamura ${ }^{3}$, Nobuhiro Saruki ${ }^{4}$, Akira Imaizumi ${ }^{5^{*}}$, Hiroshi Yamamoto ${ }^{5}$, Takashi Daimon ${ }^{6}$, Osamu Tochikubo ${ }^{7}$, Toru Mitsushima ${ }^{8}$, Minoru Yamakado ${ }^{9}$

and Hideki Kimura'

\begin{abstract}
Background: We have recently reported on the changes in plasma free amino acid (PFAA) profiles in lung cancer patients and the efficacy of a PFAA-based, multivariate discrimination index for the early detection of lung cancer. In this study, we aimed to verify the usefulness and robustness of PFAA profiling for detecting lung cancer using new test samples.

Methods: Plasma samples were collected from 171 lung cancer patients and 3849 controls without apparent cancer. PFAA levels were measured by high-performance liquid chromatography (HPLC)-electrospray ionization (ESI)-mass spectrometry (MS).

Results: High reproducibility was observed for both the change in the PFAA profiles in the lung cancer patients and the discriminating performance for lung cancer patients compared to previously reported results. Furthermore, multivariate discriminating functions obtained in previous studies clearly distinguished the lung cancer patients from the controls based on the area under the receiver-operator characteristics curve (AUC of ROC $=0.731 \sim 0.806$ ), strongly suggesting the robustness of the methodology for clinical use. Moreover, the results suggested that the combinatorial use of this classifier and tumor markers improves the clinical performance of tumor markers.
\end{abstract}

Conclusions: These findings suggest that PFAA profiling, which involves a relatively simple plasma assay and imposes a low physical burden on subjects, has great potential for improving early detection of lung cancer.

Keywords: Plasma, Amino acid, Lung cancer, Early detection

\section{Background}

Several minimally invasive, easy-to-use cancer diagnostic methods using peripheral blood samples have recently been developed to ease the physical burden on patients and to reduce cost and time [1-3]. Computer-aided systems for data mining, (e.g., using multivariate analysis) are now readily available and have shown promising results when applied to metabolic profiles for diagnostic and clinical use [4-6]. Several applications using metabolome

\footnotetext{
*Correspondence: mshingyoji@chiba-cc.jp; akira_imaizumi@ajinomoto.com 'Division of Thoracic Diseases, Chiba Cancer Center, 666-2, Nitona-cho, Chuoku, Chiba 260-8717, Japan

${ }^{5}$ Institute for Innovation, Ajinomoto, CO., Inc, 1-1, Suzuki-cho, Kawasaki-ku, Kawasaki 210-8681, Japan

Full list of author information is available at the end of the article
}

analysis based on machine learning to diagnose human cancer using peripheral blood or urine have recently been demonstrated [7-12].

Among metabolites, amino acids are one of the most suitable candidates for focused metabolomics because they are either ingested or synthesized endogenously and play essential physiological roles both as basic metabolites and metabolic regulators. To measure amino acids, plasma free amino acids (PFAAs), which are abundant in the circulation and link all organ systems, are favorable targets because PFAA profiles are influenced by metabolic variations in specific organ systems induced by specific diseases [13-18]. Furthermore, several investigators have reported changes in PFAA profiles in cancer patients, including lung cancer patients [19-27]. However, several discrepancies exist

\section{Biomed Central}


between the results of these studies due to the limited size of the data set [22].

High-throughput techniques using high-performance liquid chromatography (HPLC) - electrospray ionization (ESI)-mass spectrometry (MS) to measure amino acids with sufficient accuracy for clinical use have also recently been developed [28-31].

By combining these technologies, we recently obtained preliminary data on the efficacy of a diagnostic index based on PFAA concentrations, known as the "AminoIndex technology", which compresses multidimensional information from PFAA profiles into a single dimension and maximizes the differences between patients and controls. This technology was shown to be useful in the early detection of colorectal, breast, and lung cancers in approximately 150 samples from a single medical institute $[32,33]$. Furthermore, we also verified the efficacy and statistical robustness of this method using larger sample sizes from multiple medical institutes and developed discriminating functions to detect five types of cancer, including lung, gastric, colorectal, breast, and prostate cancer $[34,35]$. We also found that changes in PFAA profiles that were common to all types of cancer as well as those specific to individual cancers [34] .These functions are used in the "AminoIndex ${ }^{\circledR}$ Cancer Screening" service in Japan.

Lung cancer has been the leading cause of cancer death since 1998, and in Japan, >60,000 patients have died from lung cancer since 2005 [36]. Conventionally, chest X-rays and sputum cytology are used to screen for lung cancer in patients in Japan. However, neither chest $\mathrm{X}$-rays nor sputum cytology are ideal or versatile enough to detect early lung cancer. Although chest X-rays are useful for detecting peripheral lung cancer, this method is not always suitable for early detection [37]. In addition, this technique requires highly skilled technicians to achieve sufficient accuracy. Sputum cytology has been reported to be useful only for the detection of squamous cell carcinoma and is inadequate for detecting adenocarcinoma (which is the major histological type of lung cancer in Japan) or for detecting lung cancer in asymptomatic non-smokers [37].

Compared to chest X-ray and sputum cytology, a PFAAbased diagnostic method would be easier to use because it involves a relatively simple plasma assay, imposes a lower physical burden on patients and does not require advanced technical skills. Moreover, this method can also detect lung cancer regardless of cancer stage and histological type, including small cell lung cancer $[32,34,35]$.

In this study, we aimed to verify the usefulness of PFAA profiling for lung cancer detection using samples that had never been used as a data set to derive discriminating functions. As a result, highly reproducible results were observed in both the PFAA profiles and the discriminating performance of previously obtained PFAA-based, multiplex discriminant functions, suggesting the robustness of PFAA profiling for the early detection of lung cancer.

\section{Methods \\ Ethics}

The study was conducted in accordance with the Declaration of Helsinki, and the protocol was approved by the ethics committees of the Chiba Cancer Center, the Osaka Medical Center for Cancer and Cardiovascular Diseases, the Gunma Prefectural Cancer Center, the Kanagawa Health Service Association, the Kameda Medical Center Makuhari, and the Mitsui Memorial Hospital. All subjects gave their written informed consent for inclusion before participating in the study. All data were analyzed anonymously throughout the study.

\section{Subjects}

The participants in this study consisted of Japanese patients who had previously been histologically diagnosed with lung cancer at the Chiba Cancer Center $(n=171)$ between 2007 and 2009. Control subjects $(n=3849)$ without apparent cancers who were undergoing comprehensive medical examinations at the Kanagawa Health Service Association, the Kameda Medical Center Makuhari, or the Mitsui Memorial Hospital, Japan between 2008 and 2010 were recruited to participate in the study. Among the participants, 85 cancer patients (P1) and 421 gender- and age-matched controls $(\mathrm{C} 1)$ were used as the study dataset for two preliminary studies (Table 1) [32,34]. The remaining 86 cancer patients (P2) and 323 gender- and age-matched controls $(\mathrm{C} 2)$ were used as a test dataset and were not used to derive the discriminating functions in previous studies (Table 1) [32,34]. The remaining 3427 unmatched controls (C3) were also included and were not used to derive the discriminating functions in previous studies (Table 1) [32,34].

Using these subjects, four data sets were evaluated in this study. Dataset 1 includes $\mathrm{P} 1$ and $\mathrm{C} 1$, Dataset 2 includes P2 and C2, Dataset 3 includes all of the subjects involved in this study (P1, P2, C1, C2, and C3), and Dataset 4 includes all of the patients involved in this study (P1 and P2) (Table 1).

\section{Measurement of plasma amino acid concentration}

Blood samples $(5 \mathrm{ml})$ were collected from forearm veins, after overnight fasting, in tubes containing ethylenediaminetetraacetic acid (EDTA; Termo, Tokyo, Japan) and were immediately placed on ice. Plasma was prepared by centrifugation at $3,000 \mathrm{rpm}$ and $4^{\circ} \mathrm{C}$ for $15 \mathrm{~min}$ and stored at $-80^{\circ} \mathrm{C}$ until analysis. The plasma samples were deproteinized using acetonitrile at a final concentration of $80 \%$ before measurement. The amino acid concentrations in the plasma were measured by HPLC-ESI-MS followed by precolumn derivatization. The analytical 
Table 1 Demographic and clinical characteristics of the subjects

\begin{tabular}{|c|c|c|c|c|c|c|}
\hline \multirow{2}{*}{$\frac{\text { Subjects }}{\text { Subgroup }}$} & & \multicolumn{2}{|c|}{ Patients } & \multicolumn{3}{|c|}{ Controls } \\
\hline & & P1 & P2 & $\mathrm{C} 1$ & $\mathrm{C} 2$ & $\mathrm{C} 3$ \\
\hline \multirow[t]{4}{*}{ Dataset } & 1 & Used & & Used & & \\
\hline & 2 & & Used & & Used & \\
\hline & 3 & Used & Used & Used & Used & Used \\
\hline & 4 & Used & Used & & & \\
\hline \multirow[t]{2}{*}{ Number } & Total & 85 & 86 & 421 & 323 & 3104 \\
\hline & (Male, Female) & $(49,36)$ & $(68,18)$ & $(245,176)$ & $(263,60)$ & $(1898,1206)$ \\
\hline \multirow[t]{2}{*}{ Age, y } & Mean (SD) & $65.1(9.7)$ & $67.8(8.2)$ & $63.1(8.7)$ & $61.9(6.0)$ & $49.4(8.0)$ \\
\hline & Range & $30-90$ & $41-83$ & $28-86$ & $37-88$ & $23-67$ \\
\hline \multirow[t]{2}{*}{ BMI } & Mean (SD) & $22.1(3.7)$ & $22.4(3.2)$ & $22.8(3.0)$ & $23.4(2.9)$ & $23.2(3.3)$ \\
\hline & Range & $14.6 \sim 31.2$ & 15.7-34.6 & $14.2-37.1$ & 16.9-35.4 & $14.8-41.2$ \\
\hline \multirow[t]{4}{*}{ Smoking status } & Never & 26 & 18 & 222 & 139 & 1865 \\
\hline & Ex & 29 & 36 & 106 & 107 & 434 \\
\hline & Current & 29 & 29 & 57 & 62 & 695 \\
\hline & Unknown & 1 & 3 & 36 & 15 & 110 \\
\hline \multirow[t]{5}{*}{ pStage ${ }^{a}$} & । & 33 & 33 & & & \\
\hline & $\|$ & 5 & 5 & & & \\
\hline & III & 27 & 22 & & & \\
\hline & IV & 20 & 23 & & & \\
\hline & Unknown & 0 & 3 & & & \\
\hline \multirow[t]{4}{*}{ Histology } & Adenocarcinoma & 59 & 55 & & & \\
\hline & Squamous cell carcinoma & 13 & 12 & & & \\
\hline & Other NSCLC & 5 & 8 & & & \\
\hline & SCLC & 8 & 11 & & & \\
\hline
\end{tabular}

a: Cancer stages were determined according to the International Union Against Cancer TNM Classification of Malignant Tumors, 6th Edition [38].

methods used have previously been described [29-31]. Among the 20 genetically encoded amino acids, glutamate (Glu), aspartate (Asp), and cysteine (Cys) were excluded from the analysis because they are unstable in blood. Citrulline (Cit) and ornithine (Orn) were measured instead because they are relatively abundant in blood and are known to play important roles in metabolism. The following 19 amino acids were measured and analyzed: alanine (Ala), arginine (Arg), asparagine (Asn), Cit, glutamine (Gln), glycine (Gly), histidine (His), isoleucine (Ile), leucine (Leu), lysine (Lys), methionine (Met), Orn, phenylalanine (Phe), proline (Pro), serine (Ser), threonine (Thr), tryptophan (Trp), tyrosine (Tyr), and valine (Val). The concentrations of amino acids in the plasma were expressed as $\mu \mathrm{M}$ values. For analysis of the PFAA profile, two measurements were conducted for each of the 19 amino acids. The absolute concentration of each amino acid and the ratios of the amino acid concentrations expressed by the follow equation as previously described were used $[32,34]$. The concentrations of the amino acids in the plasma were expressed in $\mu \mathrm{M}$, and the ratios of the amino acid concentrations were expressed by the follow equation:

$$
X 2_{i, j}=\frac{X_{i, j}}{\sum_{k} X_{i, k}}
$$

where $X 2_{i j}$ is the ratio of the amino acid concentration of the $\mathrm{j}$-th amino acid of the $\mathrm{i}$-th subject, and $X_{i j}$ is the plasma concentration $(\mu \mathrm{M})$ of the $j$-th amino acid of the $i$-th subject.

\section{Measurement of tumor markers}

Using serum samples from lung cancer patients, the levels of the following five tumor markers were measured: CEA (chemiluminescence immunoassay, normal range $\leqq 5.0 \mathrm{ng} /$ $\mathrm{ml}$ ), CYFRA (electrochemiluminescence immunoassay, normal range $\leqq 3.5 \mathrm{ng} / \mathrm{ml}$ ), ProGRP (enzyme-linked immunoadsorbent assay, normal range $\leqq 46 \mathrm{pg} / \mathrm{ml}$ ), SCC (enzyme immunoassay, normal range $\leqq 1.5 \mathrm{ng} / \mathrm{ml}$ ), and NSE (radioimmunoassay, normal range $\leqq 10 \mathrm{ng} / \mathrm{ml}$ ) [39].

\section{Calculation of discriminant scores}

The PFAA profiles of subjects were substituted into the discriminating functions obtained from the results of 

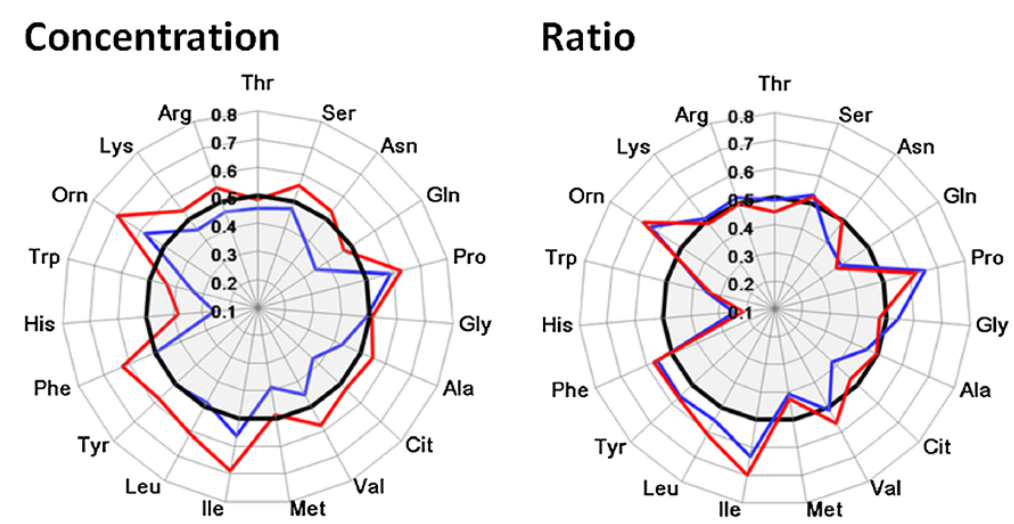

Figure 1 PFAA profiles of lung cancer patients. Axes show the AUC of the ROC for each amino acid to discriminate lung cancer patients from controls. Black bold lines indicate the point at which the $A \cup C$ of the $R O C=0.5$.

three independent preliminary studies [32,34,35]. Both Discriminant- 1 and Discriminant- 3 were logistic regression functions, whereas Discriminant- 2 was a linear discriminating function using plasma concentrations (expressed in $\mu \mathrm{M}$ ) as explanatory variables.

\section{Statistical analysis \\ Mean and SD}

The mean amino acid concentrations \pm standard deviations (SD) were calculated to determine the overall PFAA profiles for both patients and controls.

\section{Mann-Whitney U-test}

The Mann-Whitney $U$-test was used to evaluate differences in the PFAA profiles between the patient and control samples.

\section{ROC curve analysis}

Receiver-operator characteristic (ROC) curve analyses were performed to determine the abilities of both the PFAA concentrations and discriminating scores to discriminate between patients and controls. The patient labels were fixed as positive class labels. The $95 \%$ confidence interval (95\% CI) for the AUC of ROC for the discrimination of patients based on amino acid concentrations and ratios was also estimated as described by Hanley and McNeil [40].

\section{Pearson's correlation coefficients}

Pearson's correlation coefficients were calculated among three kinds of discriminant scores (obtained from Discriminant- 1, Discriminant- 2, and Discriminant3) using Dataset 3. In addition, coefficients were also calculated using stratified data (patients and controls).

\section{Determination of sensitivity}

The cutoff value for Discriminant- 3 was previously determined so that $95 \%$ specificity would be obtained [35]. The sensitivity of Discriminant- 3 was also calculated as the ratio of true positives to the summation of the true positives and false negatives. For tumor markers, sensitivities were also determined as the ratio of the number of subjects in which the marker levels were higher than the previously determined normal range to the number of measured subjects.

\section{McNemar test}

The McNemar test was performed to evaluate the improvement in sensitivities through combinatorial use of both Discriminant- 3 and the tumor markers.

\section{Software}

All of the analyses were performed using MATLAB (The Mathworks, Natick, MA) and GraphPad Prism (GraphPad Software, La Jolla, CA).

\section{Results}

\section{Characteristics of the patients and control subjects}

Table 1 summarizes the characteristics of the subjects in this study. No significant differences in body mass index (BMI) were observed between patients and matched controls (Table 1). Weight loss due to malnutrition was therefore not expected to influence the results. Although significant differences in average age were observed between the data sets, the effects appeared to be relatively minor because the absolute values of these differences were small (Table 1). Disease stages were determined according to the Sixth Edition of the International Union Against Cancer (UICC) Tumor-Node-Metastasis (TNM) Classification of Malignant Tumors [38]. The fractions of patients at each stage according to the type of cancer were as follows: $~ 40 \%$ stage I, $~ 5 \%$ stage II, $\sim 30 \%$ stage III, and $\sim 25 \%$ stage IV (Table 1).

The cancer patients were also further subdivided based on histological tumor type; approximately $65 \%$ of the patients were classified as having adenocarcinoma, 15\% 
Table 2 PFAA profiles of controls and lung cancer patients

\begin{tabular}{|c|c|c|c|c|c|c|c|c|c|c|c|c|}
\hline \multicolumn{13}{|c|}{ A. Concentration } \\
\hline \multirow{2}{*}{$\begin{array}{c}\text { Amino } \\
\text { acids }\end{array}$} & \multicolumn{2}{|c|}{ P1 } & \multicolumn{4}{|c|}{$\mathrm{C} 1$} & \multicolumn{2}{|c|}{ P2 } & \multicolumn{4}{|c|}{$\mathrm{C2}$} \\
\hline & Mean & SD & Mean & SD & $A U C^{a}$ & & Mean & SD & Mean & SD & $A U C^{a}$ & $p^{b}$ \\
\hline Thr & 115.8 & 30.8 & 118.5 & 23.6 & 0.453 & & 122.6 & 32.0 & 121.8 & 25.8 & 0.483 & \\
\hline Ser & 107.8 & 20.8 & 108.8 & 18.1 & 0.472 & & 110.7 & 20.1 & 106.7 & 17.4 & 0.560 & \\
\hline Asn & 42.6 & 7.3 & 45.2 & 6.5 & 0.383 & $p<0.001$ & 47.9 & 11.2 & 46.1 & 6.5 & 0.533 & \\
\hline Gln & 547.4 & 71.4 & 586.9 & 64.2 & 0.347 & $p<0.001$ & 577.5 & 84.1 & 587.1 & 61.5 & 0.470 & \\
\hline Pro & 141.6 & 37.1 & 132.3 & 38.4 & 0.588 & $p<0.05$ & 157.3 & 48.5 & 138.6 & 38.5 & 0.630 & $p<0.001$ \\
\hline Gly & 214.3 & 66.2 & 209.7 & 52.2 & 0.490 & & 208.0 & 63.5 & 203.5 & 47.9 & 0.511 & \\
\hline Ala & 324.2 & 84.5 & 343.1 & 74.8 & 0.428 & $<p 0.05$ & 366.5 & 99.8 & 353.5 & 69.7 & 0.550 & \\
\hline Cit & 29.0 & 8.8 & 33.0 & 7.4 & 0.367 & $p<0.001$ & 33.3 & 10.3 & 32.4 & 7.1 & 0.530 & \\
\hline Val & 215.1 & 43.1 & 220.5 & 39.7 & 0.453 & & 242.1 & 43.2 & 230.0 & 37.2 & 0.578 & $p<0.05$ \\
\hline Met & 24.2 & 5.4 & 25.8 & 4.6 & 0.388 & $p<0.01$ & 26.9 & 7.2 & 26.8 & 4.1 & 0.489 & \\
\hline lle & 64.7 & 16.2 & 60.8 & 14.4 & 0.563 & & 73.9 & 14.0 & 64.4 & 13.1 & 0.689 & $p<0.001$ \\
\hline Leu & 117.6 & 25.3 & 118.8 & 23.8 & 0.484 & & 135.2 & 26.3 & 125.2 & 21.3 & 0.610 & $p<0.01$ \\
\hline Tyr & 65.9 & 15.0 & 65.2 & 12.5 & 0.503 & & 71.9 & 15.9 & 67.3 & 10.9 & 0.579 & $p<0.05$ \\
\hline Phe & 59.5 & 9.9 & 59.6 & 9.4 & 0.496 & & 66.5 & 12.8 & 61.3 & 7.9 & 0.625 & $p<0.001$ \\
\hline $\mathrm{His}$ & 69.7 & 12.5 & 80.2 & 9.5 & 0.255 & $p<0.001$ & 74.1 & 15.8 & 81.2 & 9.4 & 0.383 & $p<0.001$ \\
\hline Trp & 51.3 & 11.0 & 57.0 & 8.8 & 0.338 & $p<0.004$ & 56.4 & 13.4 & 59.6 & 8.9 & 0.432 & \\
\hline Orn & 55.2 & 13.2 & 51.7 & 12.6 & 0.581 & $p<0.05$ & 61.7 & 16.4 & 51.7 & 10.4 & 0.696 & $p<0.001$ \\
\hline Lys & 183.9 & 32.7 & 189.1 & 30.4 & 0.450 & & 195.7 & 37.7 & 191.6 & 27.7 & 0.535 & \\
\hline Arg & 93.1 & 20.7 & 95.1 & 16.8 & 0.460 & & 100.4 & 24.7 & 96.4 & 15.1 & 0.551 & \\
\hline \multicolumn{13}{|l|}{ B. Ratio } \\
\hline Thr & 4.556 & 0.887 & 4.544 & 0.706 & 0.488 & & 4.460 & 0.749 & 4.590 & 0.781 & 0.445 & \\
\hline Ser & 4.301 & 0.765 & 4.200 & 0.669 & 0.528 & & 4.080 & 0.601 & 4.044 & 0.617 & 0.521 & \\
\hline Asn & 1.692 & 0.218 & 1.740 & 0.197 & 0.408 & $p<0.01$ & 1.749 & 0.235 & 1.742 & 0.194 & 0.494 & \\
\hline Gln & 21.800 & 2.097 & 22.661 & 2.233 & 0.383 & $p<0.001$ & 21.276 & 2.079 & 22.253 & 2.053 & 0.364 & $p<0.001$ \\
\hline Pro & 5.599 & 1.246 & 5.049 & 1.225 & 0.654 & $p<0.001$ & 5.756 & 1.504 & 5.213 & 1.211 & 0.622 & $p<0.001$ \\
\hline Gly & 8.543 & 2.566 & 8.102 & 1.994 & 0.542 & & 7.608 & 1.872 & 7.703 & 1.734 & 0.477 & \\
\hline Ala & 12.715 & 2.141 & 13.123 & 2.156 & 0.459 & & 13.253 & 2.380 & 13.321 & 2.092 & 0.499 & \\
\hline Cit & 1.150 & 0.328 & 1.278 & 0.288 & 0.378 & $p<0.001$ & 1.211 & 0.311 & 1.229 & 0.263 & 0.469 & \\
\hline Val & 8.530 & 1.294 & 8.454 & 1.109 & 0.510 & & 8.925 & 1.267 & 8.696 & 1.198 & 0.563 & \\
\hline Met & 0.955 & 0.128 & 0.990 & 0.115 & 0.407 & $p<0.01$ & 0.979 & 0.152 & 1.011 & 0.108 & 0.424 & $p<0.05$ \\
\hline lle & 2.558 & 0.519 & 2.321 & 0.405 & 0.631 & $p<0.001$ & 2.727 & 0.441 & 2.427 & 0.415 & 0.698 & $p<0.001$ \\
\hline Leu & 4.657 & 0.760 & 4.547 & 0.643 & 0.551 & & 4.986 & 0.795 & 4.731 & 0.671 & 0.607 & $p<0.01$ \\
\hline Tyr & 2.616 & 0.495 & 2.503 & 0.368 & 0.560 & & 2.644 & 0.455 & 2.543 & 0.335 & 0.567 & \\
\hline Phe & 2.374 & 0.370 & 2.295 & 0.291 & 0.557 & & 2.470 & 0.492 & 2.322 & 0.272 & 0.572 & $p<0.05$ \\
\hline His & 2.769 & 0.395 & 3.091 & 0.291 & 0.245 & $p<0.001$ & 2.702 & 0.355 & 3.074 & 0.295 & 0.213 & $p<0.001$ \\
\hline Trp & 2.034 & 0.348 & 2.197 & 0.301 & 0.351 & $p<0.001$ & 2.057 & 0.373 & 2.260 & 0.313 & 0.338 & $p<0.001$ \\
\hline Orn & 2.195 & 0.482 & 1.984 & 0.413 & 0.631 & $p<0.001$ & 2.298 & 0.674 & 1.955 & 0.353 & 0.662 & $p<0.001$ \\
\hline Lys & 7.278 & 0.809 & 7.265 & 0.834 & 0.507 & & 7.169 & 0.760 & 7.241 & 0.824 & 0.485 & \\
\hline Arg & 3.680 & 0.568 & 3.657 & 0.525 & 0.519 & & 3.650 & 0.550 & 3.645 & 0.473 & 0.494 & \\
\hline
\end{tabular}


Table 3 Three discriminating functions and amino acids used in each function

\begin{tabular}{clc}
\hline Discriminant & Amino acids incorporated into the model & Reference \\
\hline 1 & Ala, Val, lle, His, Trp, Orn & {$[32]$} \\
2 & Ser, Gln Pro, Cit, Val, lle, Phe, His, Trp, Orn & {$[34]$} \\
3 & Ser, Gln, Ala, His, Orn, Lys & {$[35]$} \\
\hline
\end{tabular}

as having squamous cell carcinoma, and $10 \%$ as having small cell lung cancer (SCLC) (Table 1).

\section{PFAA profiles of lung cancer patients}

First, the PFAA profiles of the study data set in previous studies and of the test data set, which was never used for analysis, were used to verify the changes in PFAA profiles observed in cancer patients. Interestingly, the PFAA profiles of the test data set were quite similar to those of the study data set, especially for the ratios of the amino acid concentrations (Figure 1 and Table 2), indicating that the alteration in PFAA profiles observed in cancer patients is robust. Significant increases in both the concentration and ratio of Pro and Orn and significant decreases in His were observed in both the study and test data sets compared to controls (Figure 1 and Table 2). The ratios more clearly reflected the alterations in the PFAA profile than the concentrations; the profiles of five additional amino acids were altered in the ratio data (Gln, Met, and His were decreased in patients, and Ile was increased in patients), while significant changes in concentration were detected in only one direction (Figure 1 and Table 2).

\section{Verification of multivariate discriminating functions}

We used three different discriminating functions to distinguish lung cancer patients from controls (Table 3). Discriminant 1 was derived from the PFAA profiles of cancer patients recruited from the Osaka Medical Center for Cancer and Cardiovascular Diseases and controls recruited from the Center for Multiphasic Health Testing and Services of the Mitsui Memorial Hospital [32]. Discriminant 2 and Discriminant 3 were derived from patients from the Osaka Medical Center for Cancer and Cardiovascular Diseases, the Chiba Cancer Center, the Kanagawa Cancer Center, and the Gunma Prefectural Cancer Center and controls recruited from the Center for Multiphasic Health

Table 4 AUCs of the ROC and the $95 \%$ confidential intervals $(95 \% \mathrm{Cls})$ for each model

\begin{tabular}{|c|c|c|c|c|c|c|}
\hline & \multicolumn{2}{|c|}{ Discriminant-1 } & \multicolumn{2}{|c|}{ Dinscriminant-2 } & \multicolumn{2}{|c|}{ Discriminant-3 } \\
\hline & AUC & $95 \% \mathrm{Cl}$ & $\overline{A U C}$ & $95 \% \mathrm{Cl}$ & $\overline{A U C}$ & $95 \% \mathrm{Cl}$ \\
\hline & 0.731 & $0.668-$ & 0.822 & 375 & 0.777 & 0.718 \\
\hline F & 7 & 5 & 0 & 5 & 1 & $0.700-0.823$ \\
\hline ataset 3 & 805 & $767-0.846$ & 0.806 & $0.767-0.843$ & 0.795 & $0755-083$ \\
\hline
\end{tabular}

Testing and Services of the Mitsui Memorial Hospital, the Kameda Medical Center Makuhari, and the Kanagawa Health Service Association [34,35]. Discriminant 3 is commercially used in the "AminoIndex ${ }^{\circledR}$ Cancer Screening" service in Japan (Ajinomoto, CO., Inc.) [35]. Both Discriminant 1 and Discriminant 3 were logistic regression models, whereas Discriminant 2 was a linear discriminating function. Explanatory variables used in these functions are listed in Table 3.

Three different data sets (Dataset 1, Dataset 2, and Dataset 3) were used to verify the performance of the discriminating functions (Table 4 and Figure 2). Notably, the discrimination abilities of each data set were evaluated using the AUC of the ROC of the discriminate score and were found to be $>0.7$ in all cases, indicating that the discrimination functions were both reproducible and robust using independent data sets (Figure 2, Table 4). Specifically, AUCs for the discrimination of lung cancer patients were estimated as follows: 0.731 (95\% CI: 0.668 0.794) for Dataset 1, 0.822 (95\% CI: 0.768 - 0.875) for Dataset 2, and 0.777 (95\% CI: 0.718 - 0.836) for Dataset 3 for Discriminant- 1; 0.797 (95\% CI: 0.738 - 0.856) for Dataset 1, 0.775 (95\% CI: 0.714 - 0.836) for Dataset 2, and 0.761 (95\% CI: 0.700 - 0.823) for Dataset 3 for Discriminant 2; and 0.805 (95\% CI: 0.767 - 0.846) for Dataset 1, 0.806 (95\% CI: $0.767-0.843)$ for Dataset 2, and 0.795 (95\% CI: 0.755 - 0.831) for Dataset 3 for Discriminant 3 (Figure 2, Table 4).

Selected explanatory variables partially overlapped for the discriminating functions (Table 3); therefore, the discriminant scores were highly mutually correlated as presented in Table 5. The correlation coefficients were as follows: 0.609 (Discriminant- 1 and Discriminant- 2), 0.552 (Discriminant1 and Discriminant- 3), and 0.719 (Discriminant- 2 and Discriminant- 3) for all of the data in Dataset 3. For the patients in Dataset 3 (i.e., P1 and P2), the correlation coefficients were 0.559 (Discriminant- 1 and Discriminant- 2), 0.506 (Discriminant- 1 and Discriminant- 3), and 0.686 (Discriminant- 2 and Discriminant- 3), and the correlation coefficients for the controls in Dataset 3 (i.e., C1, C2, and C3) were 0.674 (Discriminant- 1 and Discriminant- 2), 0.645 (Discriminant- 1 and Discriminant- 3), and 0.810 (Discriminant- 2 and Discriminant- 3) (Table 5).

\section{Combinatorial use of discriminating functions and tumor markers}

For further investigation of the clinical applicability of PFAA profiles, the combinatorial use of both the discriminating function from PFAA profiles as explanatory variables and existing tumor markers generally used for lung cancer detection and monitoring (CEA, CYFRA, ProGRP, SCC, and NSE) was assessed $[39,41]$. In this analysis, Dataset 4 (P1 and P2) was analyzed using discriminant scores obtained from Discriminant- 3. Subgroup analysis was also 


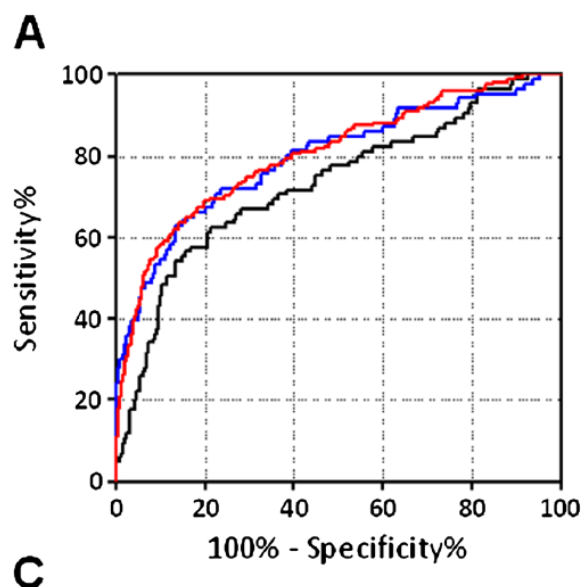

B

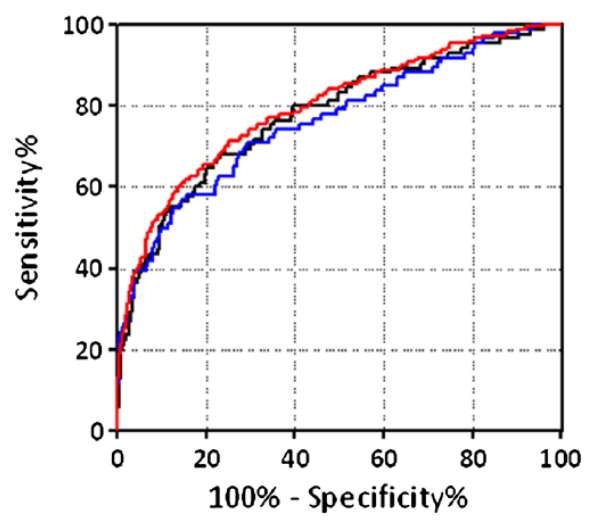

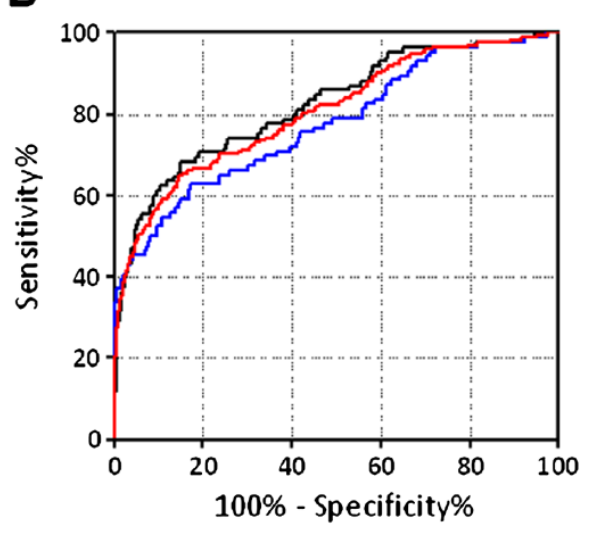

Figure 2 ROC curves of discriminating scores for each discriminating function. Black lines indicate the ROC curves of Dataset 1 , blue lines indicate those of Dataset 2, and red lines indicate those of Dataset 3.

performed using patient data stratified into cancer stages (stages I and II). For all patients, significantly higher sensitivities were observed upon combinatorial use of Discriminant- 3 and the tumor markers than upon single use of either Discriminant- 3 or the tumor markers (Figure 3). Similar results were observed among stage I and II patients using the combination of Discriminant- 3 and three tumor markers (CEA, SCC, and NSE), while no significant improvement of sensitivity was observed using Discriminant- 3 and CYFRA or ProGRP (Figure 3). These results suggest that the combinatorial use of Discriminant 3 and other tumor markers is effective for lung cancer detection and monitoring, and an increase in sensitivity was indeed confirmed (Figure 3).

Among the tumor markers, CYFRA and SCC are specific to squamous cell carcinoma (SqCC), ProGRP and NSE are specific to small cell lung cancer (SCLC), and CEA is not specific to any particular histological type of lung cancer [39]. Clinically, the combinatorial use of multiple independent tumor markers is effective for detecting lung cancer. Notably, a low correlation was observed between Discriminant 3 and the tumor markers; the correlation coefficients were 0.304 for CEA, 0.481 for
CYFRA, -0.228 for ProGRP, 0.346 for SCC, and 0.102 for NSE (data not shown).

\section{Discussion}

In the present study, we verified the usefulness of PFAA profiling for lung cancer detection using new independent samples that had never been used for previous analysis and a derivation of multivariate discriminating function(s) that could distinguish lung cancer patients from control subjects. The results were highly reproducible for the change in PFAA profiles in lung cancer patients and highly discriminatory for lung cancer patients, including those with early stage cancer. Therefore, the results strongly suggest that our method is robust enough for clinical use. Moreover, because our method is a relatively simple plasma assay and imposes minimal physical burden on subjects, our findings suggest that PFAA profiling has great potential for improving the early detection of lung cancer.

Among the three discriminating functions, several amino acids were used in more than one function. His and Orn were incorporated into all of the functions, and Ser, Gln, Ala, Val, Ile, and Trp were incorporated into 
Table 5 Correlation coefficients among the discriminant scores of Dataset 3 and its subgroups obtained from three discriminating functions

\begin{tabular}{cccc}
\hline Compared with; & All data & Patients (P1, P2) & Controls (C1, C2, C3) \\
\hline Discriminant- 1 and Discriminant- 2 & 0.609 & 0.559 & 0.674 \\
Discriminant- 1 and Discriminant- 3 & 0.552 & 0.506 & 0.645 \\
Discriminant- 2 and Discriminant- 3 & 0.719 & 0.685 & 0.810 \\
\hline
\end{tabular}

two of the three functions (Table 3) [32,34,35]. According to a comparison between the study and test data sets, plasma concentrations of Pro, Ile and Orn were higher in each data set, while the concentrations of Gln, His and Trp were lower (Figure 1 and Table 2). Among these amino acids, changes in the plasma concentrations of four amino acids (Pro, Ile, His, and Orn) were identical to the changes in amino acids in lung cancer patients in previous studies. Maeda et al. have reported that plasma concentrations of Ser, Pro, Gly, Ala, Met, Ile, Leu, Tyr, Phe, Orn, and Lys are increased and His is decreased in lung cancer patients [32]. Miyagi et al. have also reported that the plasma concentrations of Ser, Pro, Gly, Ile, and Orn are increased, whereas Gln, Cit, His, and Trp are decreased in lung cancer patients [34]. Therefore, the results strongly suggest the robustness of these three discriminating functions for the detection of lung cancer.
Moreover, Miyagi et al. have also reported that plasma levels of Gln, Trp, His, Pro, and Orn are commonly altered in cancer patients with five types of cancer (lung cancer, gastric cancer, colorectal cancer, breast cancer, and prostate cancer) [34]. Therefore, the data also strongly suggest that the changes in plasma concentrations of Pro, His, and Orn are essentially associated with carcinogenesis and cancer progression regardless of the location of the tumor.

Although tumor markers have been used extensively to detect lung cancer and estimate clinical condition, the markers are not always useful due to low specificity and insufficient sensitivity. Therefore, combinatorial use of two or more independent tumor markers is necessary for clinical utility [39]. Our results suggest that a PFAAbased diagnostic method would be a novel index to improve the insufficient clinical performance of the tumor markers. Combinatorial use of the tumor markers with

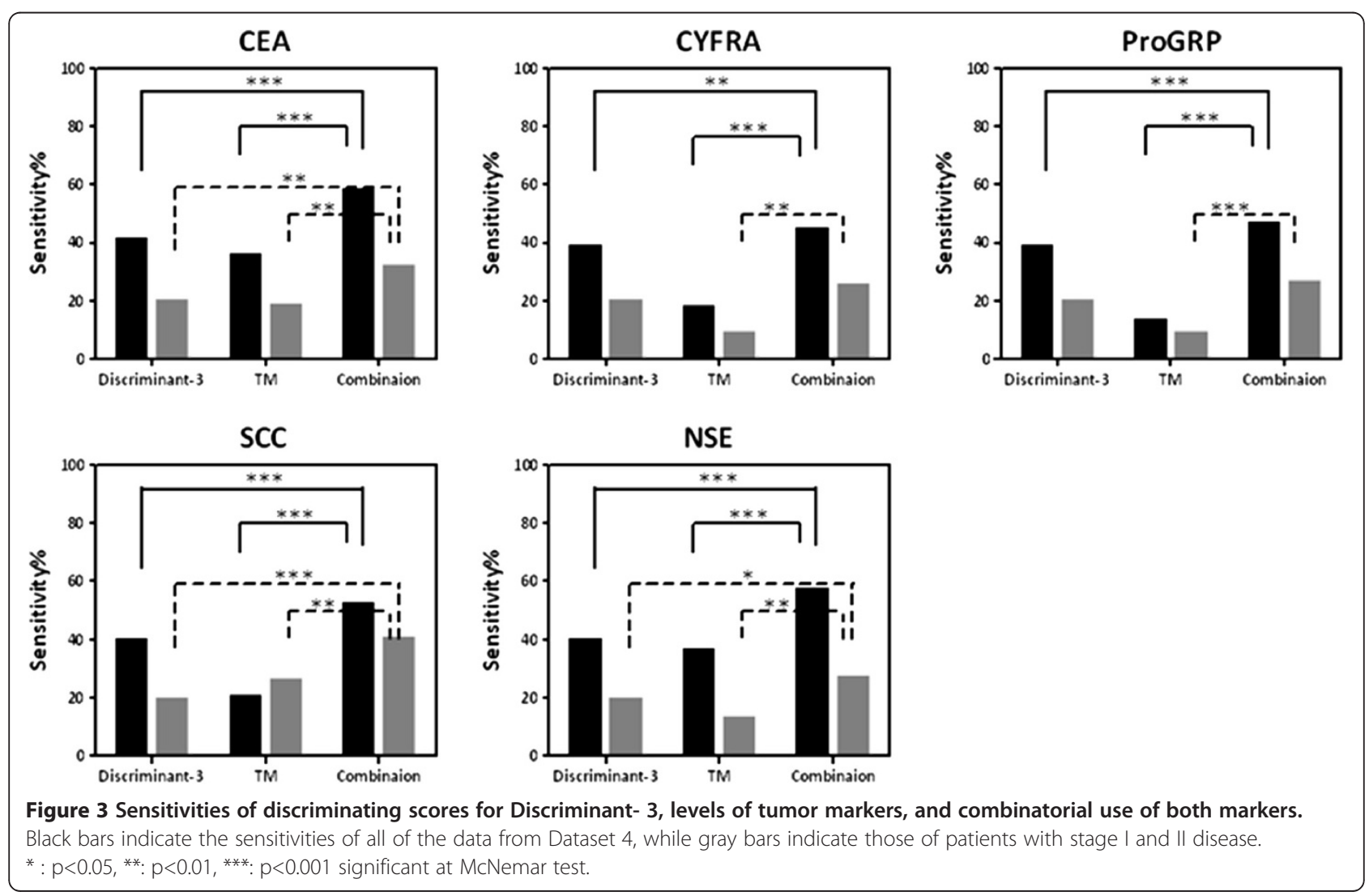


Discriminant- 3 showed higher sensitivities than any of the tumor markers generally used for lung cancer patients. Additionally, only a low correlation was observed between the discriminating function scores and the tumor marker levels, suggesting the independence of the PFAA profiles from the existing tumor markers. Miyagi et al. have suggested that the change in the PFAA profile in cancer patients reflects two aspects: metabolic changes common to many cancers and metabolic characteristics specific to each cancer [34]. Indeed, although the results were preliminary, the same study demonstrated the possibility of discriminating the cancer type. To clarify this hypothesis, testing the behavior of the discriminating function scores in lung cancer patients after surgery and chemotherapy and in those with recurrence would be necessary.

Because this study was designed as a case-control study, the results cannot be directly applied to further observation or prediction. Therefore, additional validation using a larger sample size is necessary to establish the clinical utility of our approach. Nonetheless, we believe that our results strongly suggest the clinical usefulness of the PFAA-based diagnostic method for the detection of lung cancer.

\section{Competing interest}

Takashi Daimon, Osamu Tochikubo, and Minoru Yamakado have been consultants for Ajinomoto, Co., Inc. and receive consultancy fees from Ajinomoto, CO., Inc. Akira Imaizumi and Hiroshi Yamamoto are employees of Ajinomoto, CO., Inc. Masato Shingyoji, Toshihiko lizasa, Masahiko Higashiyama, Fumio Imamura, Nobuhiro Saruki, Osamu Tochikubo, Toru Mitsushima, Minoru Yamakado, and Hideki Kimura have received research grants from Ajinomoto, CO., Inc. Masahiko Higashiyama, Fumio Imamura, Akira Imaizumi, and Hiroshi Yamamoto have applied for patents for plasma amino acid profiling using multivariate analysis as a diagnostic tool for lung cancer (WO2008/016111).

\section{Authors' contributions}

$\mathrm{Al}, \mathrm{HY}$ and $\mathrm{HK}$ designed this study. MS, TI, MH, Fl, NS, and HK coordinated the study and collected the background data on the patients. HY, OT, TM, and MY also coordinated the study and supervised the collection of control subjects. MS and Al provided data analysis and wrote the manuscript. Al and TD performed statistical analyses. All authors read and approved the final paper.

\section{Acknowledgements}

We thank Dr. Hiroshi Miyano, Mr. Takashi Yamamoto, and Ms. Naoko Kageyama for the amino acid analysis, Dr. Katsuhisa Horimoto for help with the statistical analysis, and Ms. Mariko Takasu and Ms. Tomoko Kasakura for help with data acquisition. We also thank all members of the medical staff of the Chiba Cancer Center, the Osaka Medical Center for Cancer and Cardiovascular Diseases, the Gunma Prefectural Cancer Center, the Center for Multiphasic Health Testing and Services of the Mitsui Memorial Hospital, the Kameda Medical Center Makuhari, and the Kanagawa Health Service Association for help with sample collection.

\section{Author details}

'Division of Thoracic Diseases, Chiba Cancer Center, 666-2, Nitona-cho, Chuoku, Chiba 260-8717, Japan. 'Department of Thoracic Surgery, Osaka Medical Center for Cancer and Cardiovascular Diseases, 1-3-3, Nakamichi, Higashinariku, Osaka 537-8511, Japan. ${ }^{3}$ Department of Pulmonary Oncology, Osaka Medical Center for Cancer and Cardiovascular Diseases, 1-3-3, Nakamichi, Higashinari-ku, Osaka 537-8511, Japan. ${ }^{4}$ Department of Anesthesia, Gunma Prefectural Cancer Center, 617-1, Takahayashi-nishicho, Ohta 373-8550, Japan. 5 Institute for Innovation, Ajinomoto, CO., Inc, 1-1, Suzuki-cho, Kawasaki-ku, Kawasaki 210-8681, Japan. ${ }^{6}$ Department of Biostatistics, Hyogo College of
Medicine, 1-1, Mukogawa-cho, Nishinomiya, Japan. ${ }^{7}$ Kanagawa Health Service Association, 58, Nihon-odori, Naka-ku, Yokohama 231-0021, Japan.

${ }^{8}$ Department of Gastroenterology, Kameda Medical Center Makuhari, 1-3-CD2, Nakase, Mihama-ku, Chiba 261-8501, Japan. ${ }^{9}$ Center for Multiphasic Health Testing and Services, Mitsui Memorial Hospital, 1, Kanda-izumicho, Chiyoda-ku, Tokyo 101-8643, Japan.

Received: 21 November 2012 Accepted: 7 February 2013 Published: 15 February 2013

\section{References}

1. Hunter MP, Ismail N, Zhang X, Aguda BD, Lee EJ, Yu L, Xiao T, Schafer J, Lee ML, Schmittgen TD, et al: Detection of microRNA expression in human peripheral blood microvesicles. PLoS One 2008, 3(11):e3694.

2. Roth C, Kasimir-Bauer S, Pantel K, Schwarzenbach H: Screening for circulating nucleic acids and caspase activity in the peripheral blood as potential diagnostic tools in lung cancer. Mol Oncol 2011, 5(3):281-291.

3. Roth C, Rack B, Muller V, Janni W, Pantel K, Schwarzenbach H: Circulating microRNAs as blood-based markers for patients with primary and metastatic breast cancer. Breast Canc Res 2010, 12(6):R90.

4. Chadeau-Hyam M, Ebbels TM, Brown IJ, Chan Q, Stamler J, Huang CC, Daviglus ML, Ueshima H, Zhao L, Holmes E, et al: Metabolic profiling and the metabolome-wide association study: significance level for biomarker identification. J Proteome Res 2010, 9(9):4620-4627.

5. Lee K, Hwang D, Yokoyama T, Stephanopoulos G, Stephanopoulos GN, Yarmush ML: Identification of optimal classification functions for biological sample and state discrimination from metabolic profiling data. Bioinformatics 2004, 20(6):959-969.

6. Kim Y, Koo I, Jung BH, Chung BC, Lee D: Multivariate classification of urine metabolome profiles for breast cancer diagnosis. BMC Bioinforma 2010, 11(2):S4.

7. Nolen B, Velikokhatnaya L, Marrangoni A, De Geest K, Lomakin A, Bast RC Jr, Lokshin A: Serum biomarker panels for the discrimination of benign from malignant cases in patients with an adnexal mass. Gynecol Oncol 2010, 117(3):440-445.

8. Pasikanti KK, Esuvaranathan K, Ho PC, Mahendran R, Kamaraj R, Wu QH, Chiong E, Chan EC: Noninvasive urinary metabonomic diagnosis of human bladder cancer. J Proteome Res 2010, 9(6):2988-2995.

9. Qiu Y, Cai G, Su M, Chen T, Zheng X, Xu Y, Ni Y, Zhao A, Xu LX, Cai S, et al: Serum metabolite profiling of human colorectal cancer using GC-TOFMS and UPLC-QTOFMS. J Proteome Res 2009, 8(10):4844-4850.

10. Qiu Y, Patwa TH, Xu L, Shedden K, Misek DE, Tuck M, Jin G, Ruffin MT, Turgeon DK, Synal $S$, et al: Plasma glycoprotein profiling for colorectal cancer biomarker identification by lectin glycoarray and lectin blot. J Proteome Res 2008, 7(4):1693-1703.

11. Rocha CM, Barros AS, Gil AM, Goodfellow BJ, Humpfer E, Spraul M, Carreira $I M$, Melo JB, Bernardo J, Gomes A, et al: Metabolic profiling of human lung cancer tissue by $1 \mathrm{H}$ high resolution magic angle spinning (HRMAS) NMR spectroscopy. J Proteome Res 2010, 9(1):319-332.

12. Urayama S, Zou W, Brooks K, Tolstikov V: Comprehensive mass spectrometry based metabolic profiling of blood plasma reveals potent discriminatory classifiers of pancreatic cancer. Rapid Commun Mass Spectrom 2010, 24(5):613-620.

13. Felig P, Marliss E, Ohman JL, Cahill CF Jr: Plasma amino acid levels in diabetic ketoacidosis. Diabetes 1970, 19(10):727-728.

14. Felig P, Wahren J, Raf L: Evidence of inter-organ amino-acid transport by blood cells in humans. Proc Natl Acad Sci USA 1973, 70(6):1775-1779.

15. Fischer JE, Funovics JM, Aguirre A, James JH, Keane JM, Wesdorp RI, Yoshimura N, Westman T: The role of plasma amino acids in hepatic encephalopathy. Surgery 1975, 78(3):276-290.

16. Fischer JE, Rosen HM, Ebeid AM, James JH, Keane JM, Soeters PB: The effect of normalization of plasma amino acids on hepatic encephalopathy in man. Surgery 1976, 80(1):77-91.

17. Kimura T, Noguchi Y, Shikata N, Takahashi M: Plasma amino acid analysis for diagnosis and amino acid-based metabolic networks. Curr Opin Clin Nutr Metab Care 2009, 12(1):49-53.

18. Noguchi $Y$, Zhang QW, Sugimoto T, Furuhata Y, Sakai R, Mori M, Takahashi M, Kimura T: Network analysis of plasma and tissue amino acids and the generation of an amino index for potential diagnostic use. Am J Clin Nutr 2006, 83(2):513S-519S. 
19. Cascino A, Cangiano C, Ceci F, Franchi F, Mineo T, Mulieri M, Muscaritoli M, Rossi Fanelli F: Increased plasma free tryptophan levels in human cancer: a tumor related effect? Anticancer Res 1991, 11(3):1313-1316.

20. Cascino A, Muscaritoli M, Cangiano C, Conversano L, Laviano A, Ariemma S, Meguid MM, Rossi Fanelli F: Plasma amino acid imbalance in patients with lung and breast cancer. Anticancer Res 1995, 15(2):507-510.

21. Kubota A, Meguid MM, Hitch DC: Amino acid profiles correlate diagnostically with organ site in three kinds of malignant tumors. Cancer 1992, 69(9):2343-2348.

22. Lai HS, Lee JC, Lee PH, Wang ST, Chen WJ: Plasma free amino acid profile in cancer patients. Semin Canc Biol 2005, 15(4):267-276.

23. Laviano A, Cascino A, Muscaritoli M, Fanfarillo F, Rossi Fanelli F: Tumorinduced changes in host metabolism: a possible role for free tryptophan as a marker of neoplastic disease. Adv Exp Med Biol 2003, 527:363-366.

24. Naini AB, Dickerson JW, Brown MM: Preoperative and postoperative levels of plasma protein and amino acid in esophageal and lung cancer patients. Cancer 1988, 62(2):355-360.

25. Norton JA, Gorschboth CM, Wesley RA, Burt ME, Brennan MF: Fasting plasma amino acid levels in cancer patients. Cancer 1985, 56(5):1181-1186

26. Proenza AM, Oliver J, Palou A, Roca P: Breast and lung cancer are associated with a decrease in blood cell amino acid content. J Nutr Biochem 2003, 14(3):133-138,

27. Vissers YL, Dejong CH, Luiking YC, Fearon KC, von Meyenfeldt MF, Deutz NE: Plasma arginine concentrations are reduced in cancer patients: evidence for arginine deficiency? Am J Clin Nutr 2005, 81(5):1142-1146.

28. Fontana S, Alessandro R, Barranca M, Giordano M, Corrado C, Zanella-Cleon I, Becchi M, Kohn EC, De Leo G: Comparative proteome profiling and functional analysis of chronic myelogenous leukemia cell lines. $J$ Proteome Res 2007, 6(11):4330-4342.

29. Shimbo K, Kubo S, Harada Y, Oonuki T, Yokokura T, Yoshida H, Amao M, Nakamura M, Kageyama N, Yamazaki J, et al: Automated precolumn derivatization system for analyzing physiological amino acids by liquid chromatography/mass spectrometry. Biomed Chromatogr 2009, 24(7):683-691.

30. Shimbo K, Oonuki T, Yahashi A, Hirayama K, Miyano H: Precolumn derivatization reagents for high-speed analysis of amines and amino acids in biological fluid using liquid chromatography/electrospray ionization tandem mass spectrometry. Rapid Commun Mass Spectrom 2009, 23(10):1483-1492.

31. Shimbo K, Yahashi A, Hirayama K, Nakazawa M, Miyano H: Multifunctional and highly sensitive precolumn reagents for amino acids in liquid chromatography/ tandem mass spectrometry. Anal Chem 2009, 81(13):5172-5179.

32. Maeda J, Higashiyama M, Imaizumi A, Nakayama T, Yamamoto H, Daimon T, Yamakado M, Imamura F, Kodama K: Possibility of multivariate function composed of plasma amino acid profiles as a novel screening index for non-small cell lung cancer: a case control study. BMC Canc 2010, 10(1):690.

33. Okamoto N, Miyagi Y, Chiba A, Akaike M, Shiozawa M, Imaizumi A, Yamamoto H, Ando T, Yamakado M, Tochikubo O: Diagnostic modeling with differences in plasma amino acid profiles between non-cachectic colorectal/breast cancer patients and healthy individuals. Int J Med Med Sci 2009, 1(1):1-8.

34. Miyagi $Y$, Higashiyama M, Gochi A, Akaike M, Ishikawa T, Miura T, Saruki N, Bando $E$, Kimura $H$, Imamura $F$, et al: Plasma free amino acid profiling of five types of cancer patients and its application for early detection. PLOS One 2011, 6(9):e24143.

35. Okamoto N: Cancer screening using "Aminolndex Technology". Ningen Dock 2011, 26(3):454-466

36. Asamura H, Goya T, Koshiishi Y, Sohara Y, Eguchi K, Mori M, Nakanishi Y, Tsuchiya R, Shimokata K, Inoue H, et al: A Japanese Lung Cancer Registry study: prognosis of 13,010 resected lung cancers. J Thorac Oncol 2008, 3(1):46-52.

37. Sumitani M, Takifuji N, Nanjyo S, Imahashi Y, Kiyota H, Takeda K, Yamamoto $\mathrm{R}$, Tada $\mathrm{H}$ : Clinical relevance of sputum cytology and chest $X$-ray in patients with suspected lung tumors. Intern Med 2008, 47(13):1199-1205.

38. Sobin L, Wittekind C: TNM Classification of Malignant Tumours, Sixth Edition. New York: Wiley-Liss: 2002
39. Ando S, Kimura H, Iwai N, Kakizawa K, Shima M, Ando M: The significance of tumour markers as an indication for mediastinoscopy in non-small cell lung cancer. Respirology 2003, 8(2):163-167.

40. Hanley JA, McNeil BJ: The meaning and use of the area under a receiver operating characteristic (ROC) curve. Radiology 1982, 143(1):29-36.

41. Molina R, Auge JM, Filella X, Vinolas N, Alicarte J, Domingo JM, Ballesta AM: Pro-gastrin-releasing peptide (proGRP) in patients with benign and malignant diseases: comparison with CEA, SCC, CYFRA 21-1 and NSE in patients with lung cancer. Anticancer Res 2005, 25(3A):1773-1778.

doi:10.1186/1471-2407-13-77

Cite this article as: Shingyoji et al:: The significance and robustness of a plasma free amino acid (PFAA) profile-based multiplex function for detecting lung cancer. BMC Cancer 2013 13:77.

\section{Submit your next manuscript to BioMed Central and take full advantage of:}

- Convenient online submission

- Thorough peer review

- No space constraints or color figure charges

- Immediate publication on acceptance

- Inclusion in PubMed, CAS, Scopus and Google Scholar

- Research which is freely available for redistribution

Submit your manuscript at www.biomedcentral.com/submit
C) Biomed Central 